\title{
An iron-clad interaction
}

DOI:

10.1038/nrmicro1628
Five arenaviruses are known to cause acute viral haemorrhagic fevers in humans, each of which features on the Category A Pathogen List established by the US Centres for Disease Control and Prevention. Reporting in Nature, Hyeryun Choe and colleagues demonstrate that transferrin receptor 1 (TfR1) is the host cellular receptor for the four New World haemorrhagic fever arenaviruses - Junin, Machupo, Guanarito and Sabia.

Arenaviruses are enveloped, single-stranded RNA viruses that can be classified into two antigenically and geographically distinct groups, the Old World arenaviruses and the New World arenaviruses. Previous studies had identified $\alpha$-dystroglycan as the cellular receptor that allowed Lassa virus - an Old World arenavirus — to invade host cells but also showed that this receptor was not used by the New World arenaviruses. As the viral glycoprotein (GP) of these viruses is required for cell entry, Radoshitzky et al. focused on the interaction between the GP expressed by the Machupo virus and cells known to be permissive to Machupo virus infection. Biochemical and mass spectrometric analysis of this interaction revealed a specific, high affinity association with the cellular protein TfR1. The authors also showed that expression of TfR1 in hamster cell lines efficiently enhanced the entry of retroviruses expressing the GP of Machupo virus. Expression of transferrin receptor 2 in the same cell lines did not promote virus infection. To determine whether TfR 1 was the host cell receptor for the other New World arenaviruses, the authors constructed viruses pseudotyped with the GPs of the Guanarito and Junin viruses and showed that TfR1 was required in both cases for successful infection. Finally, to confirm the role of TfR 1 as the common cellular receptor for all four New World arenaviruses, an anti-TfR1 antibody was shown to efficiently inhibit infection in all cases.

As TfR 1 is normally involved in cellular iron transport, Radoshitzky et al. also investigated the effect of iron on the arenavirus infection process. When the cell culture medium was depleted of iron, which is known to upregulate the expression of TfR1, there was a significant enhancement

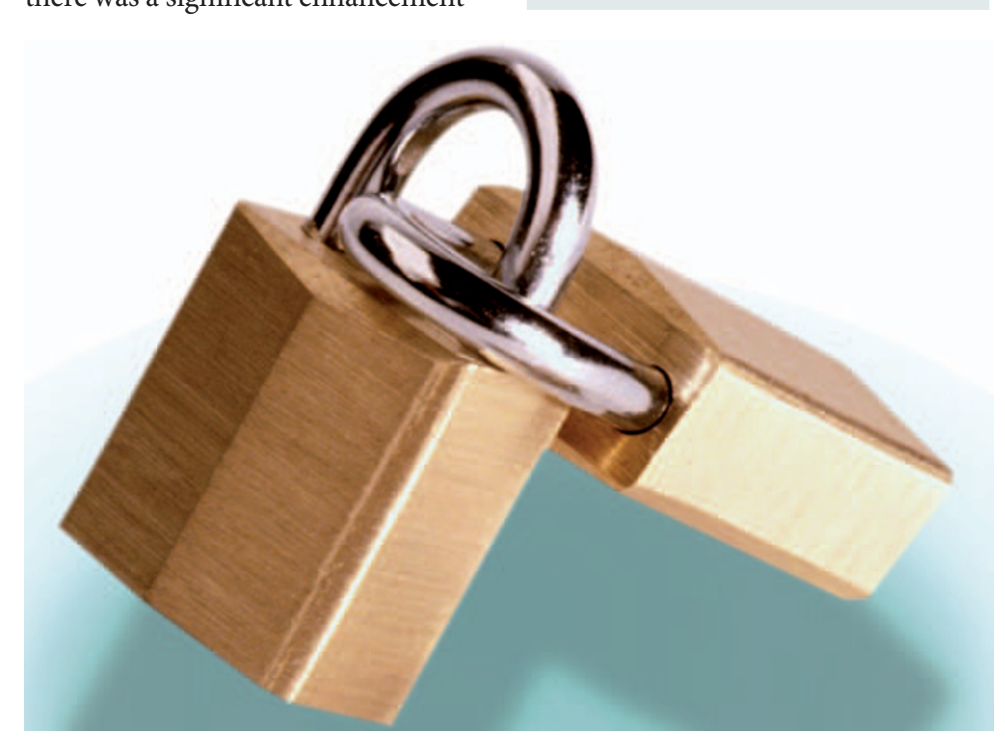

in the efficiency of infection by both the Junin and Machupo viruses. Conversely, supplementation of the medium with iron (and the subsequent down regulation of TfR1 expression) inhibited Junin and Machupo virus infection.

Together these data clearly indicate that TfR1 is the cellular receptor for New World haemorrhagic fever arenaviruses. The role of iron in this process, the possible consequences of iron deficiency, and the therapeutic potential of a humanized anti-TfR1 antibody are all important directions for future research.

David O'Connell

\section{ORIGINAL RESEARCH PAPER}

Radoshitzky, S. R. et al. Transferrin receptor 1 is a cellular receptor for New World haemorrhagic fever arenaviruses. Nature 07 February 2007 (doi:10.1038/nature05539) 